\title{
Gene expression profiling reveals two separate mechanisms regulating apoptosis in rectal carcinomas in vivo
}

\author{
Elza C. de Bruin - Simone van de Pas • \\ Cornelis J. H. van de Velde · J. Han J. M. van Krieken • \\ Lucy T. C. Peltenburg - Corrie A. M. Marijnen • \\ Jan Paul Medema
}

Published online: 3 July 2007

(C) Springer Science+Business Media, LLC 2007

\begin{abstract}
The level of apoptosis in rectal carcinomas of patients treated by surgery only predicts local failure; patients with intrinsically high-apoptotic tumors develop less local recurrences than patients with low levels of apoptosis. To identify genes involved in this intrinsic apoptotic pro-
\end{abstract}

Electronic supplementary material The online version of this article (doi: 10.1007/s10495-007-0088-2) contains supplementary material, which is available to authorized users.

E. C. de Bruin - S. van de Pas - L. T. C. Peltenburg ·

C. A. M. Marijnen

Department of Clinical Oncology, Leiden University Medical

Center, Leiden, The Netherlands

E. C. de Bruin · J. P. Medema ( $ه)$

Laboratory for Experimental Oncology and Radiobiology,

Academic Medical Center (AMC), Meibergdreef 9, 1105 AZ

Amsterdam, The Netherlands

e-mail: j.p.medema@amc.nl

Present Address:

S. van de Pas

Department of Anatomy and Embryology, Leiden University

Medical Center, Leiden, The Netherlands

C. J. H. van de Velde

Department of Surgery, Leiden University Medical Center,

Leiden, The Netherlands

J. H. J. M. van Krieken

Department of Pathology, Radboud University Nijmegen

Medical Center, Nijmegen, The Netherlands

Present Address:

L. T. C. Peltenburg

Crucell Holland B.V., Leiden, The Netherlands

C. A. M. Marijnen

Department of Radiotherapy, Netherlands Cancer Institute,

Amsterdam, The Netherlands cess in vivo, 47 rectal tumors with known apoptotic phenotype (24 low- and 23 high-apoptotic) were analyzed by oligonucleotide microarray technology. We identified several genes differentially expressed between low- and high-apoptotic tumors. Unsupervised clustering of the tumors based on expression levels of these genes separated the low-apoptotic from the high-apoptotic tumors, indicating a gene expression-dependent regulation. In addition, this clustering revealed two subgroups of high-apoptotic tumors. One high-apoptotic subgroup showed subtle differences in mRNA and protein expression of the known apoptotic regulators BAX, cIAP2 and ARC compared to the low-apoptotic tumors. The other subgroup of highapoptotic tumors showed high expression of immune-related genes; predominantly HLA class II and chemokines, but also HLA class I and interferon-inducible genes were highly expressed. Immunohistochemistry revealed HLADR expression in epithelial tumor cells in $70 \%$ of these high-apoptotic tumors. The expression data suggest that high levels of apoptosis in rectal carcinoma patients can be the result of either slightly altered expression of known pro- and anti-apoptotic genes or high expression of immune-related genes.

Keywords Rectal cancer - Apoptosis - Gene expression profiling

\section{Introduction}

Apoptosis is an important intrinsic characteristic of rectal tumors. High amounts of apoptotic tumor cells correlate with a reduced local recurrence risk in rectal cancer patients who were treated by surgery only $[1,2]$. From the literature, it is known that the apoptotic process can be 
regulated by two main pathways: the death-receptor pathway and the mitochondrial pathway. The death-receptor pathway is induced via engagement of death receptors, such as CD95, by their ligands. Receptor polymerization leads to the recruitment of the death-inducing signaling complex, resulting in the activation of caspase- 8 . The mitochondrial pathway is activated during cellular stress, such as DNA damage or oxidative stress, via the release of cytochrome $c$ from the mitochondrion resulting in the activation of caspase-9. This cytochrome $c$ release is controlled by the pro- and anti-apoptotic members of the Bcl-2 family.

Deregulation of these apoptotic pathways can contribute to a number of human diseases, like neurodegenerative disorders and cancer. During progression from normal cell to tumor cell and consequent metastasis, the cells are exposed to a range of apoptotic stresses. Cells that acquire mutations that suppress apoptosis will have a selective growth advantage and will expand. Such anti-apoptotic mutations can therefore result in therapy-resistant tumors and are thought to contribute to treatment failure. For rectal cancer, several studies have evaluated the role of apoptosis for the sensitivity of tumors to anti-cancer treatment. Tumors with high levels of intrinsic apoptosis were indeed more likely to regress upon neo-adjuvant radiochemotherapy than tumors where apoptosis was low (reviewed by Smith et al. [3]), indicating that these intrinsically highapoptotic tumors are more sensitive to apoptotic triggers. Since this sensitivity can be regulated by the Bcl- 2 family of proteins, use of the pro-apoptotic Bax and the antiapoptotic Bcl-2 proteins as predictive markers for response to neo-adjuvant therapy has been studied. Two recent publications indeed suggest a role for Bax in therapy response $[4,5]$, but most studies found no correlation between the expression of $\mathrm{Bax}$ or $\mathrm{Bcl}-2$ and tumor downstaging or clinical outcome of the patients after radioand/or chemotherapy [3].

Interestingly, rectal cancer patients treated only by surgery had a relatively low local recurrence rate when the tumor intrinsic level of apoptosis was high. Because this intrinsic apoptosis occurred in the absence of neo-adjuvant treatment, it is important to know which proteins are responsible for the low or high levels of intrinsic apoptosis in vivo. The objective of our study was therefore to identify genes involved in the induction and/or progression of intrinsic apoptosis in human tumors. Since the process of apoptosis can be regulated by multiple proteins (pro- and anti-apoptotic proteins) and at several levels in the pathways (up- and downstream of the mitochondria), identification of apoptosis regulators requires assessment of the expression of a large number of genes. Oligonucleotide microarray analysis allowed us to assess such large numbers of genes simultaneously. We used this technique to analyze rectal tumors with known apoptotic phenotype, available from a prospective multicenter trial with standardized surgery and pathologic examination [6], and compared the expression profiles of low- versus highapoptotic tumors. The genome-wide information reported here contributes to our understanding of apoptosis in vivo.

\section{Materials and methods}

Patients and tumor samples

Tumor samples were obtained at time of surgery from rectal cancer patients participating in the Dutch TME trial [6]. A total amount of 47 freshly frozen non-irradiated tumors were selected with a clear distinction in their apoptotic phenotype (lowest versus highest quartiles of apoptosis levels) as previously determined by immunohistochemical staining with the M30 antibody [2]; 24 tumors had low levels of apoptosis $(<5$ M30-positive cells/ $\mathrm{mm}^{2}$ tumor epithelium) and 23 tumors had high levels of apoptosis (> 25 M30-positive cells $/ \mathrm{mm}^{2}$ tumor epithelium). There was no significant difference between these low- and high-apoptotic tumors with respect to clinicopathologic characteristics such as gender, age, TNM stage or tumor differentiation status.

RNA isolation and microarray procedure

RNA was extracted as described in detail elsewhere [2] from macrodissected samples containing at least $60 \%$ tumor epithelium, as determined by haematoxylin and eosin (HE)-stained frozen sections. Quality of total RNA was assessed with lab-on-a-chips on the Agilent 2100 Bioanalyzer (Agilent Technologies, Palo Alto, California). All samples were shown to be free of DNA contamination and for each sample the ratio $28 \mathrm{~S} / 18 \mathrm{~S}$ was $>1.5$. Amplifications of mRNA and dye couplings were performed using Ambion's Amino Allyl MessageAmp ${ }^{\mathrm{TM}}$ kit and protocol (Ambion Inc., Austin, TX). Per microarray experiment, 2.0- $\mu \mathrm{g}$ aliquots of RNA were amplified, and 5.0- $\mu \mathrm{g}$ aliquots of amino allyl-labeled aRNA was coupled to CyDye Post Labelling Reactive Dyes (Ambion Inc.): Cy5 for tumor samples, and $\mathrm{Cy} 3$ for reference sample, consisting of pooled RNAs isolated from five normal rectum samples and five colorectal cancer cell lines (HCT116, LS411N, SW480, HCT15 and Caco2).

\section{Oligonucleotide microarrays}

About 2.0- $\mu \mathrm{g}$ aliquots of labeled sample RNA were mixed with equal amounts of labeled reference RNA and fragmented using RNA Fragmentation Reagents and protocol 
(Ambion Inc). About $20 \mu \mathrm{g}$ yeast tRNA (Invitrogen, Carlsbad, CA) and $20 \mu \mathrm{g}$ polyadenylic acid (Sigma-Aldrich, St. Louis, MO) were added and mixtures were heated at $100^{\circ} \mathrm{C}$ for $1 \mathrm{~min}$, placed on ice for $2 \mathrm{~min}$ and at $42^{\circ} \mathrm{C}$ for 5 min. Preheated $2 \times$ hybridization buffer $(50 \%$ formamide, $10 \times$ SSC, $0.01 \%$ SDS) was added just before hybridization at $42^{\circ} \mathrm{C} \mathrm{o} / \mathrm{n}$ onto the Human Genome Oligo Set Version 3.0 arrays, manufactured at the Central Microarray Facility (CMF) of the Netherlands Cancer Institute. Information about this oligo set (e.g. oligonucleotide sequence, gene symbol, protein description, EnsembleID and GO-term) is available at the website of Operon (http://www.omad.operon.com). Protocols, GeneID list and information about arrays are available at the website of the CMF (http://www.microarrays.nki.nl). Of each slide, two images were scanned using the Agilent G2565BA Microarray Scanner (Agilent Technologies), at 50 and $100 \%$ gain intensities. Spot intensities were extracted from the scanned images with Genepix 5.1 (Axon, Baden, Switzerland). Spots with aberrant shapes were flagged by the software and checked manually.

\section{Computational and statistical analysis}

Raw intensity data (.gpr files) were directly loaded into the $\mathrm{R}$ environment (http://www.r-project.org). For normalization of the arrays and identification of differentially expressed genes, the Limma (linear models for microarray data) package of Bioconductor (http://www.bioconductor.org) was applied. Control spots and spots with more than $10 \%$ saturation, a diameter smaller than $60 \mu \mathrm{m}$ or signal intensity less than 150 above background were excluded from the analyses. Data were corrected for local background (method normexp) and normalized within arrays and between arrays (method quantile). The spots of the low and high intensity scans were normalized separately. Duplicate experiments were done for 10 different tumor samples, showing Pearson correlation coefficients ranging from 0.86 to 0.92 .

Statistically significant differences in the staining of the oligos were assessed using a moderate empirical Bayes test statistics [7] available through the Limma software package. The $B$-value is the log-odds that a gene is differently expressed. The obtained $p$-values were corrected for multiple testing using the False Discovery Rate method [8]. Oligos with $B$-values $\geq 0.5$ and corrected $p$-values $\leq 0.05$ were considered statistically significant. These oligos were grouped into biological categories using the Gene Ontology (GO) terms provided by the manufacturer.

Unsupervised hierarchical clustering (with 'unweighted average' and 'correlation') was done with Spotfire Decisionsite $^{\mathrm{TM}}$ (Somerville, MA, USA) using the normalized $\log 2$ Cy3/Cy5-data.
The Gene Ontology Tree Machine (GOTM; http:// www.genereg.ornl.gov/gotm) tool was used to identify biological processes that were significantly over-represented among the oligos found to be differentially expressed when compared with the list of all oligos on the array [9]. For these GOTM-analyses we used the EnsembleIDs corresponding to the oligonucleotide sequences on the array as gene identifiers.

\section{Quantitative RT-PCR (qPCR)}

For first strand cDNA synthesis, $1.5-\mu \mathrm{g}$ aliquots of total RNA were reverse transcribed using oligo(dT)-primers and M-MLV Reverse Transcriptase (Promega Corporation, WI, USA), according to manufacturer's instructions. Total RNA was available from 35 tumor samples that were analyzed by microarray analysis.

The real-time PCR primer sets were designed by $\mathrm{Su}$ perArray (SuperArray Bioscience Corporation, MD, USA). Real-time amplifications were done according to manufacturer's protocol using $\mathrm{RT}^{2}$ Real-Time ${ }^{\mathrm{TM}}$ SYBR Green/ Fluorescein PCR Master Mix (SuperArray Bioscience Corporation). Reactions were run on an iCycler iQ Realtime Detection System (Bio-Rad Laboratories, CA, USA). A melt curve was generated at the end of each run in order to determine the specificity of the product. A calibration curve, consisting of several dilutions of cDNA of the reference used for the microarray procedure, was included for each gene to allow calculation of the relative starting quantities. These relative starting quantities were used in the normalization and statistical analyses.

Expression levels were normalized to three genes (CPSF6, GAPDH and HPRT1) to acquire a reliable normalization of the qPCR data. These genes showed the least variation between all samples as determined by the geNorm programme [10]. This geNorm method provides a normalization factor per gene, representative of the amount of mRNA in each sample.

To compare the qPCR data with the microarray data, the qPCR data were $\log _{2}$ transformed and Pearson correlation coefficients were determined using SPSS statistical software (version 12.0 for Windows, SPSS, Inc., Chicago, IL).

\section{Immunohistochemisty}

Stainings were performed on 4- $\mu \mathrm{m}$ sections using the following antibodies: anti-Bax (N-20) at 1:200 (Santa Cruz Biotechnology, Santa Cruz, CA), anti-cIAP2 (AF817) at 1:200 (R\&D Systems, Minneapolis, MN), anti-ARC (CT) at 1:2,000 (ProSci Inc., Poway, CA), anti-HLA-A (HCA2) at 1:100 (kindly provided by Dr. J. Neefjes) and anti-HLADR (clone LN3; Signet Laboratories Inc., Dedham, MA). For Bax, cIAP2 and HLA-A the staining procedure was 
done as described previously [5, 11, 12]. For ARC and HLA-DR we used the following protocol: tissues had been deparaffinised and endogenous peroxidise activity had been blocked by $0.3 \%$ hydrogen peroxide in methanol for $30 \mathrm{~min}$. Antigen retrieval was performed by boiling for $10 \mathrm{~min}$ in $0.01 \mathrm{M}$ citrate buffer ( $\mathrm{pH} 6.0$ ). Subsequently, sections were preincubated for 30 min with $20 \%$ normal goat serum in PBS/1\% BSA. After overnight incubation with the primary antibody sections were incubated for $1 \mathrm{~h}$ with biotinylated goat-anti-mouse or goat-anti-rabbit (1:200, DakoCytomation, Glostrup, Denmark) followed by incubation with streptavidin complexed with biotinylated peroxidase (DakoCytomation), and developed in 3,3-diamino-benzidine (DakoCytomation). Finally, sections were counterstained with Mayer's haematoxylin. As negative control, the primary antibody in $\mathrm{PBS} / 1 \%$ BSA was replaced by PBS alone. The results presented for each antibody are based on the analysis of tissues from at least 4 individuals per group.

\section{Results}

Genes differentially expressed in low- and highapoptotic rectal tumors

Previously we have reported that rectal cancer patients with high levels of apoptosis in their tumors develop less frequent local recurrences than patients with lower levels of apoptosis [2]. Because this high level of apoptosis occurred in the absence of neo-adjuvant treatment, it is thought to be an intrinsic characteristic of the tumors, suggesting regulation at the level of gene expression. To identify genes of which the expression correlates with the level of intrinsic apoptosis in vivo, 47 non-irradiated rectal tumors with known apoptotic phenotype were analyzed by oligo microarray technology. We selected 24 low-apoptotic and 23 high-apoptotic tumors ( $<5$ and $>25$ apoptotic cells/ $\mathrm{mm}^{2}$ tumor epithelium, respectively) with equal distributions of TNM stages, differentiation status and amounts of stromal tissue. Comparing these low- and high-apoptotic tumors, we identified 85 oligos representing 60 annotated genes differentially expressed at statistically significant levels (Supplementary Table 1). Several genes code for proteins that have been described to play a role in apoptosis regulation, such as ARC [13-15], DDAH2 [16], STC2 [17] and Stefin A [18]. GO terms were used to classify the genes with known functions into biological processes, and showed that most of the identified genes code for proteins that are associated with antigen processing and presentation, metabolism, proliferation and transcription (Fig. 1).

Despite the clear differences in apoptotic levels between the two tumor groups, which also correlate with different clinical outcomes, we observed a relatively minor change in expression of the identified oligos (Supplementary Table 1). This observation could imply that intrinsic apoptosis is regulated in a gene-expression independent manner or that genetic differences are obscured by the fact that multiple subgroups exist within the high- or low-apoptotic tumors. To analyze whether different subgroups exist, unsupervised clustering of the tumors based on the 85 identified oligos was performed (Fig. 2A). As expected, a high concordance of clustering was observed for the apoptotic phenotype; most low-apoptotic tumors were clustered to the left and most high-apoptotic tumors were clustered to the right side of the tree. Interestingly, two subgroups were visible within the group of high-apoptotic tumors. Further analysis confirmed this observation and revealed that unsupervised clustering of only the high-apoptotic tumors divided these tumors into two clear groups, denoted clusters A and B (Fig. 2B).

The existence of two distinct high-apoptotic clusters suggests that two different gene expression profiles were related with the process of apoptosis in vivo. Therefore, we determined the differences between clusters $\mathrm{A}$ and B by comparing the microarray data of the two subclusters. This analysis revealed 255 oligos that were differentially expressed at significant levels (Supplementary Table 2). A high amount of these oligos code for proteins involved with the immune system (defense response, immune response and antigen processing and presentation). GOTM analysis was done to compare the list of genes represented by the

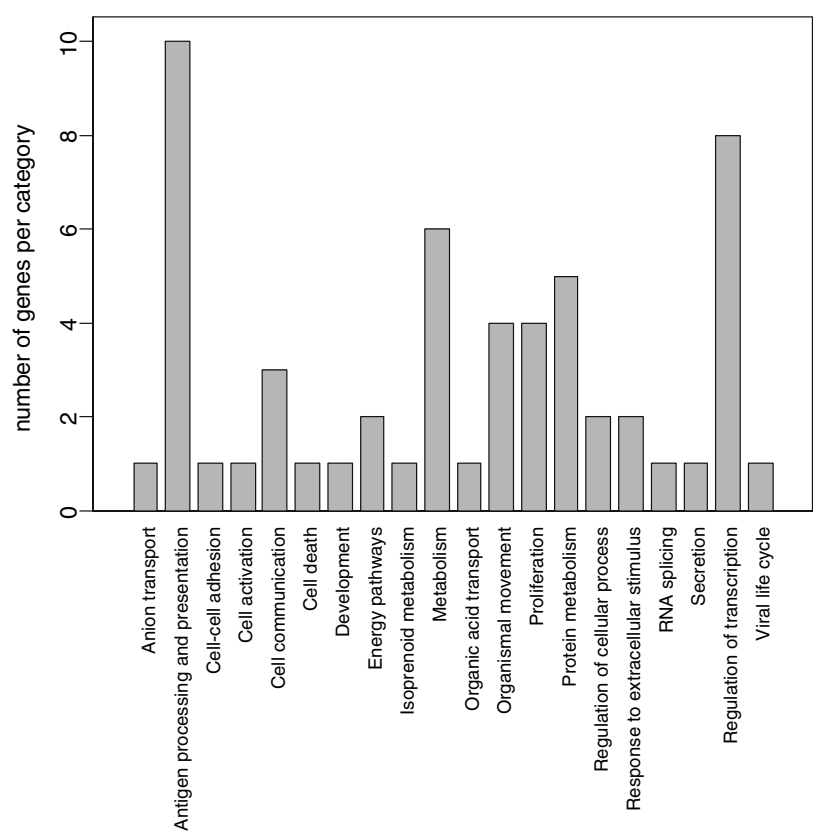

Fig. 1 Clustering of genes in biological processes. Genes represented by the 86 identified oligos were clustered in biological categories according to their GO term 


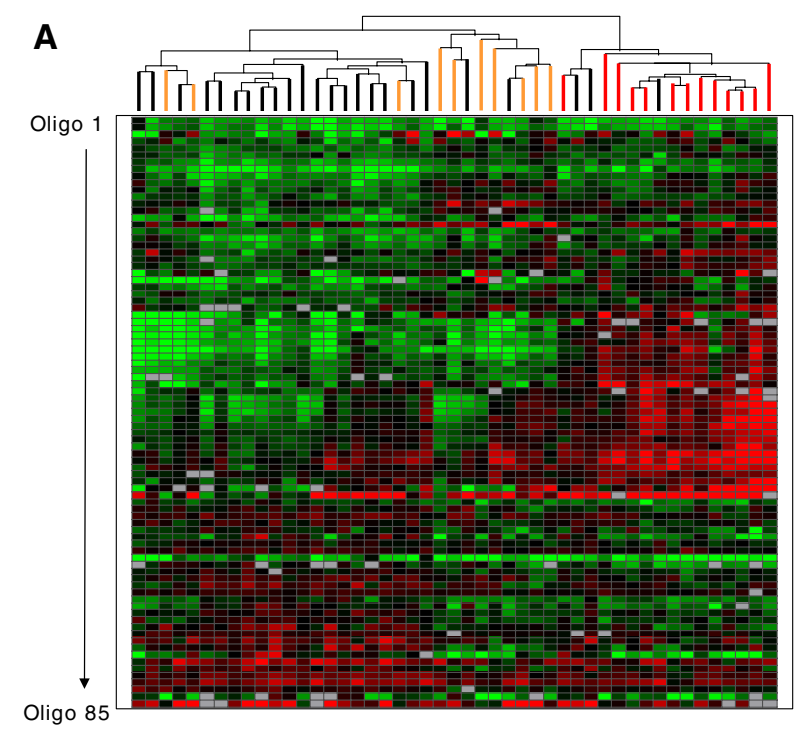

B

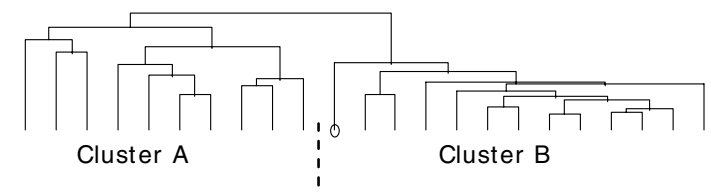

Fig. 2 Unsupervised clustering of low- and high-apoptotic tumors. (A) Unsupervised clustering of all tumors based on the microarray data of the 86 discriminating oligos. Each column represents a tumor sample and each row represents an oligo (listed in Supplementary Table 1 in same order as represented in this clustering); green indicates a lower and red indicates a higher presence of the oligo in the tumors compared with the reference. The tree above indicates the correlation between tumors; a black line for the low-apoptotic tumors, and a colored line for the high-apoptotic tumors. (B) Unsupervised clustering of only the high-apoptotic tumors separated the tumors into two subgroups, denoted as cluster A and cluster B. Cluster A is compiled of the tumors which were indicated in orange, and cluster B of the tumors which were indiacted in red in (A). Note that the tumors of cluster A are close to the low-apoptotic tumors

255 discriminating oligos with all oligos on the array of which the expression profile could be evaluated. These immune-related biological processes were indeed significantly enriched in the list of genes that were differentially expressed. Interestingly, the immune-related genes were all highly expressed in cluster B. During tumor selection, we excluded differences in amounts of stroma between the high- and low-apoptotic tumors. But since we used macrodissected samples, the immune-related expression profile of cluster B could result from difference in the amount of stromal cells between clusters A and B. We therefore reevaluated HE-stained slides of the macrodissected tumors and found no substantial differences in morphology or in the amount of stroma between the tumor clusters (Fig. 3). These data exclude that the immune-related gene expression profile of cluster B results from simply more stromal cells.
The gene expression data therefore indicate that different sets of genes are involved in the regulation of apoptosis in clusters $\mathrm{A}$ and $\mathrm{B}$.

\section{High-apoptotic phenotype of Cluster A}

Five of the genes differentially expressed in the highapoptotic clusters are related to cell death on basis of their GO term (Supplementary Table 2). Two of these have been described to play an important role in the mitochondrial pathway of apoptosis: BAX and cIAP2. We first focussed our study on these genes and validated their expression levels by qPCR. The qPCR data correlated significantly with the microarray data (Table 1) and confirmed higher expression of BAX and lower expression of cIAP2 in cluster A compared with cluster B (Fig. 4A).

In our previous analyses comparing all high-apoptotic tumors with the low-apoptotic tumors, BAX and cIAP2 were on average expressed at similar levels (fold difference between the high- and low-apoptotic tumors was 0.9 for BAX and 1.1 for cIAP2, both not statistically significant). Interestingly, the high-apoptotic tumors can be divided into two separate clusters of which cluster A showed higher BAX and lower cIAP2 expression when compared with cluster B. In addition, we observed significant lower cIAP2 expression levels and a trend towards higher BAX expression in the high-apoptotic tumors of cluster A than in the low-apoptotic tumors. These data strengthen the significance of dividing the high-apoptotic tumors into two subgroups.

To further analyze the high-apoptotic phenotype of cluster A, the microarray data of these tumors were compared with the low-apoptotic tumors. Surprisingly, this analysis revealed only five oligos, indicating a low number of differentially expressed genes (Supplementary Table 3). Genetic differences between these two groups of tumors are thus very small. This might imply an apoptosis regulation independent of gene expression levels. Nonetheless, one of the identified oligos represented the anti-apoptotic protein ARC. This protein was reported to bind Bax, and to prevent the release of cytochrome $c$ [13]. The microarray data show that ARC was expressed at significantly lower levels in the high-apoptotic cluster A when compared to the low-apoptotic tumors. Validation of the microarray data by qPCR confirmed the lower ARC expression in the tumors of cluster $\mathrm{A}$ as compared to the low-apoptotic tumors (Fig. 4A).

The differential expression of BAX, cIAP2 and ARC between the tumor clusters was evaluated on protein level by immunohistochemistry. Differences in staining intensities confirmed the mRNA data; tumors belonging to cluster A stained Bax with higher and CIAP2 with lower intensities then cluster B and low-apoptotic tumors. ARC was also lower expression in the high-apoptotic cluster A and B 

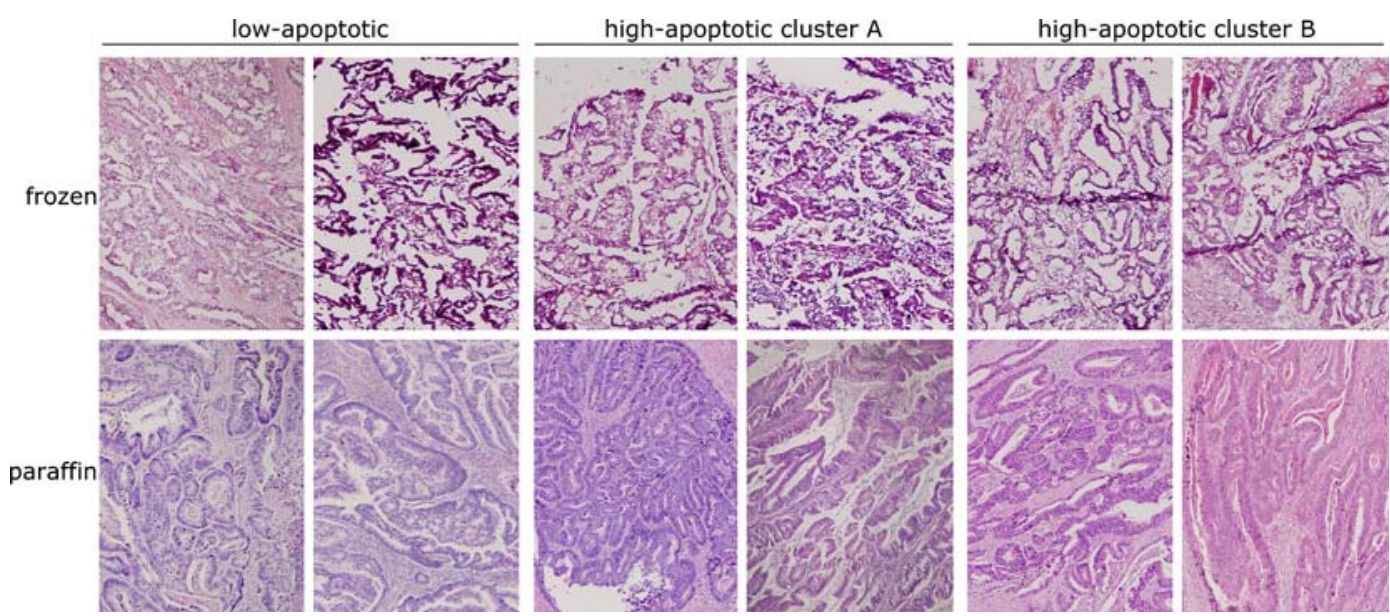

Fig. 3 Representative photomicrographs of HE-stained tumor tissues. Frozen tissues are shown in the upper panel, corresponding paraffin-embedded tissues in the lower panel. For each cluster (low-

Table 1 Correlation between qPCR and microarray data

\begin{tabular}{llc}
\hline Gene & Correlation & $p$-value \\
\hline BAX & 0.61 & $<0.001$ \\
cIAP2 & 0.58 & 0.01 \\
ARC & 0.69 & $<0.001$ \\
CXCL9 & 0.72 & $<0.001$ \\
CCL4 & 0.91 & $<0.001$ \\
CCL18 & 0.94 & $<0.001$ \\
HLA-DR & 0.92 & $<0.001$ \\
HLA-A & 0.70 & $<0.001$ \\
TAP1 & 0.69 & $<0.001$
\end{tabular}

Pearson correlation coefficients were calculated with $\log 2$-transformed qPCR and microarray data

when compared with the low-apoptotic tumors (Fig. 4B and Supplementary Fig. 1).

Overall, the high-apoptotic phenotype of the tumors belonging to cluster A might be explained by gene expression-independent regulation, or might be the result of lower expression of the anti-apoptotic ARC and cIAP2 in combination with a slightly higher expression of the proapoptotic BAX.

High-apoptotic phenotype of cluster B

The high-apoptotic tumors of cluster B formed a separate group in the unsupervised clustering analysis (Fig. 2A), suggesting a distinct gene expression profile. By comparing the gene expression profiles of these high-apoptotic tumors with the low-apoptotic tumors, we identified 322 oligos, representing 217 annotated genes differentially expressed (Supplementary Table 4). Interestingly, $37 \%$ of the oligos apoptotic, high-apoptotic cluster A, high-apoptotic cluster B) two representative photomicrographs are shown

had a 2-fold change or more in expression, indicating a substantial difference in genetic background of these groups of tumors.

GOTM analysis with this set of 322 oligos revealed a significant enrichment of immune-related categories. These categories were previously identified when cluster B was compared with cluster A, suggesting that these categories are specific for the high-apoptotic tumors of cluster B.

We focussed our study on the genes with low $p$-values and high fold-changes in their expression. A large proportion of these genes coded for immune-related proteins, such as chemokines and HLA class II and class I proteins. The expressions of the chemokines CXCL9, CCL4 and CCL18 and the antigen presenting-related proteins HLADR, HLA-A and TAP1 were validated by qPCR. The qPCR data correlated significantly with the microarray data (Table 1) and confirmed the high expression of these genes in the high-apoptotic tumors of cluster B when compared with the low-apoptotic tumors, and also with the highapoptotic tumors of cluster A (Fig. 5A). Immunohistochemistry for HLA class I and HLA class II proteins revealed no difference in staining intensity of tumor cells for HLA-A. Intriguingly, $70 \%$ of the tumors belonging to cluster B showed positive staining for HLA-DR in epithelial tumor cells, whereas low-apoptotic or cluster A tumors stained negative for HLA-DR on the epithelial tumor cells (Fig. 5B and Supplementary Fig. 2). Next to HLA-DR, several other interferon-gamma-inducible genes showed high gene expression in cluster B. Since interferongamma is produced and secreted by activated immune cells, these data suggest that the high-apoptotic phenotype of cluster $\mathrm{B}$, in contrast to cluster $\mathrm{A}$, is most likely the result of an immunologic control of these tumors. 
Fig. 4 Expression of the apoptotic genes BAX, cIAP2 and ARC. (A) Normalized relative mRNA expression levels in the groups of lowapoptotic tumors (LA), highapoptotic tumors belonging to cluster A (HA-A) or belonging to cluster B (HA-B) are represented as mean \pm SEM. $p$ values of the difference in expression level between these groups are indicated above. (B) Representative immunohistochemical stainings for Bax, cIAP2 and Arc of lowapoptotic tumors (LA), highapoptotic tumors belonging to cluster A (HA-A) or cluster B (HA-B)
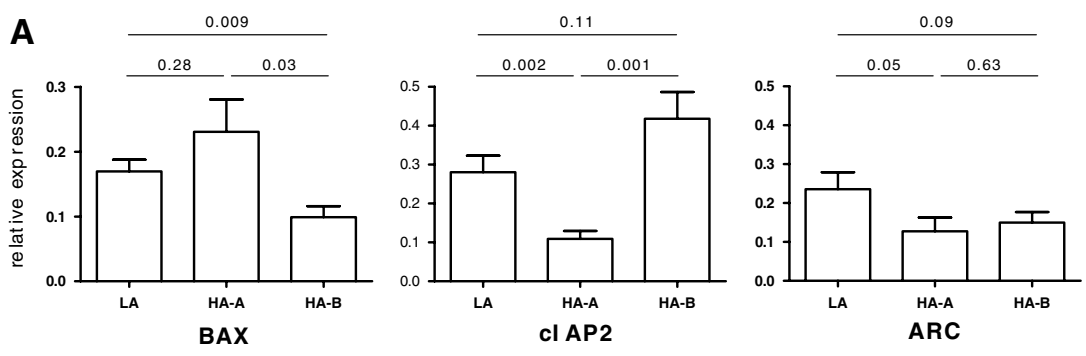

B

LA

HA-A
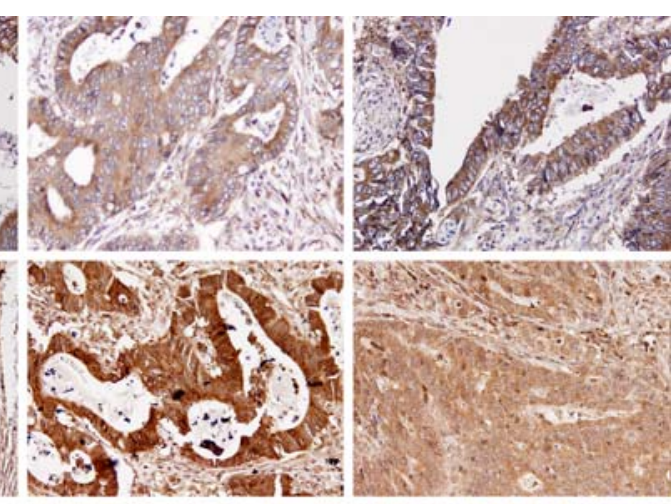

clAP2
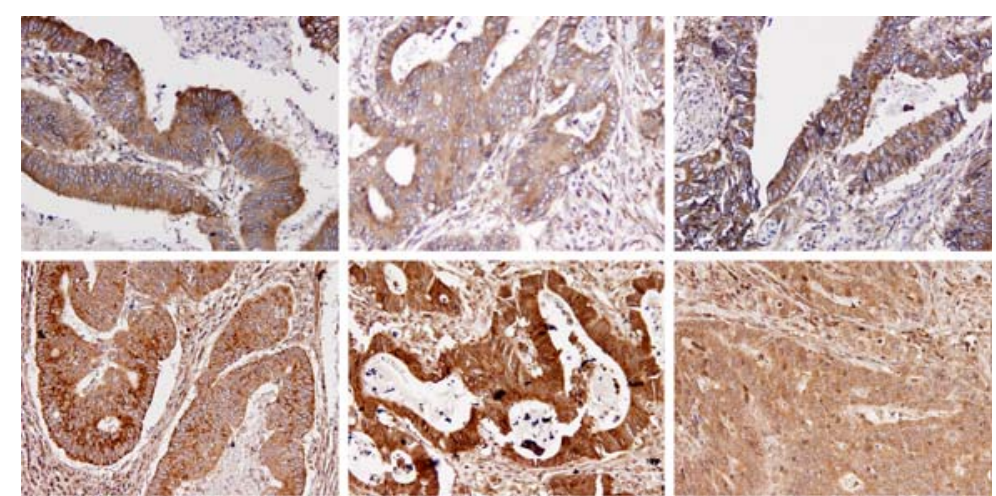

BAX
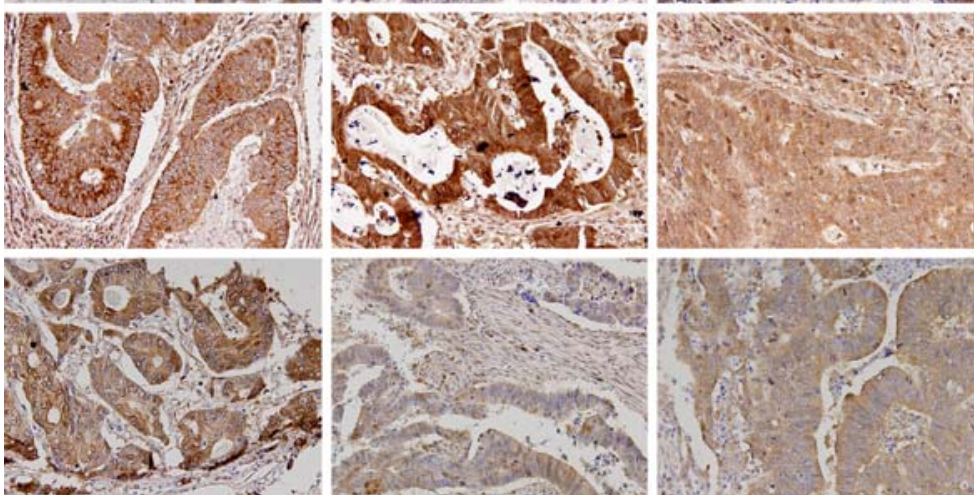

\section{Discussion}

In this study we describe the identification of genes which are differentially expressed in low- versus high-apoptotic rectal tumors. Since the rectal cancer patients were treated by surgery only, the identified genes are related to the tumor-intrinsic level of apoptosis in vivo, previously described to predict local recurrences [1, 2]. Importantly, unsupervised clustering analysis identified two clusters of high-apoptotic tumors, suggesting two distinct mechanisms regulating apoptosis in vivo. We therefore analyzed these two clusters of high-apoptotic tumors separately.

The differences in gene expression between one of the high-apoptotic clusters (cluster A) and the low-apoptotic tumors were subtle; these high-apoptotic tumors clustered close to the low-apoptotic tumors in the unsupervised clustering and a very low amount of genes were identified as differentially expressed at statistically significant levels. The high-apoptotic phenotype of these tumors might therefore, at least to some extent, be explained by geneexpression independent regulation, such as distinct localization of proteins (e.g. mitochondria versus cytosol), and inactivation or activation of proteins via phosphorylation (Bad) or cleavage (caspases). Another explanation for the low amount of differentially expressed genes can be the fact that we used strict cut-off values for significance for each single oligo in order to minimize the number of falsepositive genes. After correcting for multiple hypotheses testing, hardly any individual gene meets the threshold for statistical significance when the genetic differences are modest relative to the background noise. In that case, differences in expression of individual genes might not reach significant levels, but a combination of small differences in expression of genes belonging to the same pathway can have profound biological consequences [19].

More in-depth analyses on the known apoptotic regulators BAX, ARC and cIAP2 revealed small differences in mRNA expression among the three tumor groups, which were confirmed on protein level. The pro-apoptotic protein Bax plays an important role in the permeabilization of the mitochondrial membrane, leading to release of cytochrome $c$ into the cytosol. Cytosolic cytochrome $c$, together with APAF-1, activates caspase- 9 and subsequently caspase- 3 . The highest Bax expression was observed in cluster A of the intrinsically high-apoptotic tumors. In accordance with our results, recent clinical studies have shown that high Bax protein expression (in pretreatment biopsies) was associated with improved local control for rectal cancer patients $[4,5]$, or improved survival for colorectal carcinoma patients treated by surgery only [20]. Furthermore, 
Fig. 5 Expression of immunerelated genes. (A) Normalized relative mRNA expression levels in the groups of lowapoptotic tumors (LA), highapoptotic tumors belonging to cluster A (HA-A) or belonging to cluster B (HA-B) are represented as mean \pm SEM for the genes CXCL9, CCL4, CCL18, HLA-DR, HLA-A and TAP1. $p$-values of the difference in expression level between these groups are indicated. (B) Representative immunohistochemical stainings for HLA-A and HLA-DR of low-apoptotic tumors (LA), high-apoptotic tumors belonging to cluster A (HA-A) or cluster B (HA-B)


in vitro experiments have shown a role for Bax in the apoptotic response of colorectal cancer cells to multiple death stimuli [21, 22]. Tumors with high Bax expression in vivo might therefore be more susceptible to apoptotic triggers. In addition, the high-apoptotic tumors of cluster A demonstrated a relatively low expression of the antiapoptotic ARC and cIAP2. ARC exerts its anti-apoptotic function by interfering with Bax. In doing so, it prevents mitochondrial permeabilization and the release of cytochrome $c$ [13]. The anti-apoptotic cIAP2 belongs to the family of Inhibitor of apoptotis proteins (IAPs) that is suggested to prevent apoptosis (at least partially) due to binding to caspases, thereby inhibiting the caspase activity $[23,24]$. The cumulative effects of a slight higher expression of Bax and lower expression of ARC and cIAP2 might thus be responsible for the high-apoptotic phenotype of the tumors belonging to cluster A.

Intriguingly, the high-apoptotic tumors belonging to cluster B expressed high levels of genes that code for proteins involved in the immune response. Several publications showed that important signaling events occur between tumor cells and the surrounding stromal and infiltrating cells [25-27]. Microdissection of only tumor epithelial cells could thus lead to substantial loss of information regarding these mechanisms. We recently published that macrodissection provides more reliable data for the expression of tumor epithelial genes than laser microdissection [28]. For those reasons, we used macrodissected samples, containing stromal components. This approach resulted in the identification of a high expression of immune-related genes in the tumors of cluster B. This high expression in cluster B is probably not an effect of more stroma. We previously published that the contribution of stromal mRNA to the total amount of mRNA isolated from macrodissected tumors is minor [28], and we did not observe large differences in the amount of stroma between the tumors. In agreement, immunohistochemistry using HLA-DR, Bax and cIAP2 confirms that the differential expression results from epithelial tumor cells. Although the amounts of stroma were equally distributed, the composition of the stromal compartment can vary between the clusters. In mouse models, induced expression of HLA-DR in epithelial tumor cells enhances the infiltration of dendritic cells and anti-tumor T-cell responses [29, 30]. The high expression of chemokines in cluster B tumors might thus result from tumor infiltrating immune cells, pointing to 
a phenotypic difference between the clusters. The observation that multiple interferon-gamma-induced genes were higher expressed in these high-apoptotic tumors (e.g. TRIM22, IFI30, STAT1, IRF1, INDO) suggest that these tumors indeed mount an immune response, leading to interferon secretion and induction of interferon-responsive genes. Interferon-gamma can induce apoptosis in tumor cells directly, and also via enhancement of the immune response [31]. Our data therefore strongly suggest that these patients with intrinsically high-apoptotic tumors mount an immune response towards the tumor cells, thereby controlling tumor recurrences. Pages and coworkers recently published a role for the adaptive immune response in preventing recurrences in colorectal cancer patients $[32,33]$. Moreover, we previously published that the amount of intratumoral macrophages, NK- and T-cells correlated with a reduced local recurrence rates in rectal cancer patients [34], again indicating an immune-mediated control of rectal cancer.

\section{Conclusion}

Microarray analysis on intrinsically low- and high-apoptotic tumors identified two separate groups of high-apoptotic rectal tumors. One group demonstrated subtle differences in expression of known pro- and anti-apoptotic proteins involved in the mitochondrial pathway. Because the expression differences are small, gene independent regulation of apoptosis might play a role as well. The other group of high-apoptotic tumors showed high expression of immune-related genes, suggesting a host's immune response towards the tumor cells. Since rectal cancer patients with intrinsically high-apoptotic tumors have a better prognosis, this information may provide insight in the development of novel therapeutic regimens.

Acknowledgements This study was financially supported by the Dutch Cancer Society: RUL 2002-2733. We thank J. Oosting (Department of Pathology, Leiden University Medical Center) for providing the R-script for limma analyses, and P. Eilers (Department of Medical Statistics, Leiden University Medical Center) for advice regarding the clustering analyses. We are indebted to $\mathrm{T}$. van Wezel (Department of Pathology, Leiden University Medical Center) for advice regarding the qPCR experiments and to H. Morreau (Department of Pathology) for pathological evaluation of the macrodissection procedure. We are grateful to J. Neefjes (Department of Tumor Biology, the Netherlands Cancer Institute, Amsterdam) for kindly providing the monoclonal HCA2 antibody.

\section{References}

1. Adell GC, Zhang H, Evertsson S, Sun XF, Stal OH, Nordenskjold BA (2001) Apoptosis in rectal carcinoma: prognosis and recurrence after preoperative radiotherapy. Cancer 91:1870-1875
2. de Bruin EC, van de Velde CJ, van de Pas S et al (2006) Prognostic value of apoptosis in rectal cancer patients of the dutch total mesorectal excision trial: radiotherapy is redundant in intrinsically high-apoptotic tumors. Clin Cancer Res 12:64326436

3. Smith FM, Reynolds JV, Miller N, Stephens RB, Kennedy MJ (2006) Pathological and molecular predictors of the response of rectal cancer to neoadjuvant radiochemotherapy. Eur J Surg Oncol 32:55-64

4. Chang HJ, Jung KH, Kim DY et al (2005) Bax, a predictive marker for therapeutic response to preoperative chemoradiotherapy in patients with rectal carcinoma. Hum Pathol 36:364371

5. Nehls O, Okech T, Hsieh CJ et al (2005) Low BAX protein expression correlates with disease recurrence in preoperatively irradiated rectal carcinoma. Int J Radiat Oncol Biol Phys 61:8591

6. Kapiteijn E, Marijnen CA, Nagtegaal ID et al (2001) Preoperative radiotherapy combined with total mesorectal excision for resectable rectal cancer. N Engl J Med 345:638-646

7. Smyth GK (2004) Linear models and empirical bayes methods for assessing differential expression in microarray experiments. Stat Appl Genet Mol Biol 3:Article3

8. Benjamini Y, Hochberg Y (1995) Controlling the false discovery rate: a practical and powerful approach to multiple testing. J R Stat Soc (Series B) Stat Methodol 57:289-300

9. Zhang B, Schmoyer D, Kirov S, Snoddy J (2004) GOTree Machine (GOTM): a web-based platform for interpreting sets of interesting genes using Gene Ontology hierarchies. BMC Bioinformatics $5: 16$

10. Vandesompele J, De Preter K, Pattyn F et al (2002) Accurate normalization of real-time quantitative RT-PCR data by geometric averaging of multiple internal control genes. Genome Biol 3:RESEARCH0034

11. Vischioni B, van der Valk P, Span SW, Kruyt FA, Rodriguez JA, Giaccone G (2006) Expression and localization of inhibitor of apoptosis proteins in normal human tissues. Hum Pathol 37:78-86

12. Menon AG, Morreau H, Tollenaar RA et al (2002) Down-regulation of HLA-A expression correlates with a better prognosis in colorectal cancer patients. Lab Invest 82:1725-1733

13. Gustafsson AB, Tsai JG, Logue SE, Crow MT, Gottlieb RA (2004) Apoptosis repressor with caspase recruitment domain protects against cell death by interfering with Bax activation. $\mathbf{J}$ Biol Chem 279:21233-21238

14. Jo DG, Jun JI, Chang JW et al (2004) Calcium binding of ARC mediates regulation of caspase 8 and cell death. Mol Cell Biol 24:9763-9770

15. Neuss M, Monticone R, Lundberg MS, Chesley AT, Fleck E, Crow MT (2001) The apoptotic regulatory protein ARC (apoptosis repressor with caspase recruitment domain) prevents oxidant stress-mediated cell death by preserving mitochondrial function. J Biol Chem 276:33915-33922

16. Bruneel A, Labas V, Mailloux A et al (2005) Proteomics of human umbilical vein endothelial cells applied to etoposide-induced apoptosis. Proteomics 5:3876-3884

17. Ito D, Walker JR, Thompson CS et al (2004) Characterization of stanniocalcin 2, a novel target of the mammalian unfolded protein response with cytoprotective properties. Mol Cell Biol 24:94569469

18. Jones B, Roberts PJ, Faubion WA, Kominami E, Gores GJ (1998) Cystatin A expression reduces bile salt-induced apoptosis in a rat hepatoma cell line. Am J Physiol 275:G723-G730

19. Subramanian A, Tamayo P, Mootha VK et al (2005) Gene set enrichment analysis: a knowledge-based approach for interpreting genome-wide expression profiles. Proc Natl Acad Sci USA 102:15545-15550 
20. Schelwies K, Sturm I, Grabowski P et al (2002) Analysis of p53/ $\mathrm{BAX}$ in primary colorectal carcinoma: low BAX protein expression is a negative prognostic factor in UICC stage III tumors. Int J Cancer 99:589-596

21. Theodorakis P, Lomonosova E, Chinnadurai G (2002) Critical requirement of BAX for manifestation of apoptosis induced by multiple stimuli in human epithelial cancer cells. Cancer Res 62:3373-3376

22. Zhang L, Yu J, Park BH, Kinzler KW, Vogelstein B (2000) Role of BAX in the apoptotic response to anticancer agents. Science 290:989-992

23. Roy N, Deveraux QL, Takahashi R, Salvesen GS, Reed JC (1997) The c-IAP-1 and c-IAP-2 proteins are direct inhibitors of specific caspases. EMBO J 16:6914-6925

24. Huang H, Joazeiro CA, Bonfoco E, Kamada S, Leverson JD, Hunter T (2000) The inhibitor of apoptosis, cIAP2, functions as a ubiquitin-protein ligase and promotes in vitro monoubiquitination of caspases 3 and 7. J Biol Chem 275:26661-26664

25. Liotta LA, Kohn EC (2001) The microenvironment of the tumour-host interface. Nature 411:375-379

26. Hunter K, Welch DR, Liu ET (2003) Genetic background is an important determinant of metastatic potential. Nat Genet 34:2324

27. de Visser KE, Eichten A, Coussens LM (2006) Paradoxical roles of the immune system during cancer development. Nat Rev Cancer 6:24-37
28. de Bruin EC, van de Pas S, Lips EH et al (2005) Macrodissection versus microdissection of rectal carcinoma: minor influence of stroma cells to tumor cell gene expression profiles. BMC Genomics 6:142

29. Meazza R, Comes A, Orengo AM, Ferrini S, Accolla RS (2003) Tumor rejection by gene transfer of the MHC class II transactivator in murine mammary adenocarcinoma cells. Eur J Immunol 33:1183-1192

30. Mortara L, Castellani P, Meazza R et al (2006) CIITA-induced MHC class II expression in mammary adenocarcinoma leads to a Th1 polarization of the tumor microenvironment, tumor rejection, and specific antitumor memory. Clin Cancer Res 12:3435-3443

31. Chawla-Sarkar M, Lindner DJ, Liu YF et al (2003) Apoptosis and interferons: role of interferon-stimulated genes as mediators of apoptosis. Apoptosis 8:237-249

32. Galon J, Costes A, Sanchez-Cabo F et al (2006) Type, density, and location of immune cells within human colorectal tumors predict clinical outcome. Science 313:1960-1964

33. Pages F, Berger A, Camus $M$ et al (2005) Effector memory $T$ cells, early metastasis, and survival in colorectal cancer. N Engl J Med 353:2654-2666

34. Nagtegaal ID, Marijnen CA, Kranenbarg EK et al (2001) Local and distant recurrences in rectal cancer patients are predicted by the nonspecific immune response; specific immune response has only a systemic effect - a histopathological and immunohistochemical study. BMC Cancer 1:7 